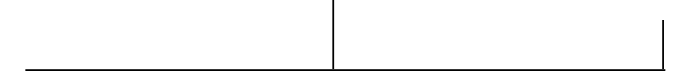

Rev. Latinoam. Psicopat. Fund., São Paulo, v. 11, n. 1, p. 29-38, março 2008

\title{
Reacciones emocionales de los niños hospitalizados con quemaduras, así como de sus familiares
}

Gabriel Zárate Guerrero

\begin{abstract}
Los accidentes por quemaduras comportan un fuerte carácter traumático; este es aún más severo cuando el accidentado es un niño. El presente texto da cuenta del trabajo de acompañamiento psicológico y psicoanalítico desarrollado en la Unidad de Quemados del Hospital Civil de Guadalajara. Se presentan los aspectos teóricos y metodológicos que sirven de base a la intervención, pudiendo constatar la importancia de la relación transferencial, de los aspectos lúdicos, de la expresión libre de los afectos y del trabajo de equipo como factores reductores del impacto traumático del accidente.
\end{abstract}

Palabras clave: Trauma psíquico, dolor, transferencia, juego 
Las reacciones emocionales que son objeto de nuestra investigación han tenido su seguimiento en la "Unidad de Niños con Quemaduras" del Hospital Civil Juan I. Menchaca, de Guadalajara Jalisco. Hemos desarrollado el Programa de Atención Psicológica de Niños con Quemaduras, coordinado por la Mtra. Leticia Ana María Pérez Cortés, desde el año 2000, en el piso 7 de dicho Hospital. Este programa, y la Unidad en general, nacieron de una serie de convenios interinstitucionales, entre los cuales destaca la colaboración con el Hospital Shriners de Galveston Texas y la Fundación Micho y Mau.

Nuestra participación con la Unidad surge a partir de una investigación que se ha propuesto identificar y conceptualizar las circunstancias y los recursos psíquicos de que disponen los niños ante un accidente por quemaduras, considerando que se trata, en particular, de sujetos en estructuración psíquica. Así, hemos articulado ese trabajo alrededor de tres objetivos: 1) identificar la dinámica propia y los elementos en juego en la conflictiva psicológica que viven los niños que han sufrido accidentes con quemaduras, así como su familia; 2) conceptualizar los efectos que dicho accidente pudieran tener respecto del proceso de constitución psíquica del niño; y, por último, 3) valorar los efectos terapéuticos de la intervención psicológica y psicoanalítica respecto de la reducción de secuelas de traumas psíquicos en estos niños y en sus familias a partir del accidente.

Como sabemos, los accidentes graves por quemaduras, además de constituir un atentado a la integridad física y corporal de los sujetos que los sufren, representan una experiencia de gran potencialidad traumática debido al alto grado de dolor, a la destrucción de tejidos, órganos o miembros, así como a las alteraciones de la imagen corporal, de la sensibilidad de la piel y de la experiencia emocional general que conllevan.

Cuando las quemaduras graves son sufridas en edad temprana, la adecuada constitución psíquica de la persona puede verse afectada. El trabajo psicológico y psicoterapéutico, así como el estudio emocional de personas que han vivido estos accidentes en la niñez, si bien permite sopesar lo que ocurre psicológicamente en esas circunstancias, también aporta elementos no sólo para entender como ayudar a reducir el carácter traumático de la experiencia, sino también para la comprensión del proceso universal de la constitución psíquica y de las diversas vicisitudes por las que pasa la constitución del sujeto humano en la infancia.

Las quemaduras son un tipo de lesión traumática corporal causada por agentes térmicos, eléctricos, químicos o radiactivos. Las quemaduras se 
clasifican en "de primer grado", "de segundo grado" y "de tercer grado". Las primeras dañan la epidermis y producen irritación de la piel, enrojecimiento y hasta ampollas. Las segundas dañan la epidermis y la dermis produciendo necrosis en tejidos internos. Las terceras son las más profundas y pueden afectar y destruir incluso huesos, nervios y órganos internos. Las quemaduras de primer y segundo grados, cuando abarcan una amplia superficie del cuerpo, pero sobre todo las de tercer grado, constituyen experiencias de gran dolor, de daño a la integridad corporal y de gran potencialidad traumática.

Interesados por las intervenciones psicológicas en los ámbitos hospitalarios e institucionales, hemos considerado dentro del psicoanálisis, las nociones de "envoltura psíquica", "yo piel" y "contenimiento". Fundamentos que nos permiten conceptualizar las vicisitudes de la constitución psíquica y narcisista de la persona, la manera como se producen los traumas psíquicos propios del accidente por quemaduras y la forma como opera la transferencia en el trabajo psicológico y psicoanalítico de acompañamiento.

Es obligado para nosotros considerar de entrada los términos de "reacción" y "emoción". La primera es una noción que nos remite al modelo del arco reflejo. Sabemos que Freud tomó este modelo simplificado para complejizarlo y para considerar los mecanismos indispensables de operación que deberían estar presentes en un aparato psíquico sometido a los embates de los estímulos y de las pulsiones. Aquí el par de elementos básicos que lo constituyen son los de acción y de reacción, los de estímulo y de respuesta.

En efecto, para Freud (1895), el contacto con la realidad no es un proceso pasivo de recepción de estímulos. El aparato psíquico lanza, de tiempo en tiempo, antenas que salen al encuentro de percepciones del mundo exterior, anticipándose así a calificar de significativas algunas experiencias más que otras. Ello nos lleva a concebir la "reacción" no solamente como engendrada por aquel estímulo específico, sino como un proceso más complejo que rescata de algún modo la historia de la persona que la produce. En el caso de los traumas psíquicos, sin embargo, ocurre una especie de corto circuito en la recuperación de esa experiencia previa. En estos casos hay un aflujo de estimulación de tan grandes proporciones que el aparato psíquico no es capaz de anticipar su recepción y su respuesta. Por ello, en el caso de los accidentes por quemaduras, en donde se generan traumas psíquicos, las reacciones emocionales que ahí se producen deberán ser objeto de atención, dado su carácter de ser vivencias no adecuadamente tramitadas en lo que respecta a la asimilación de la experiencia.

El término de "emoción", por su parte, denota una vivencia cargada de afecto, ya sea que ese afecto pueda asociarse a una representación consciente o bien que sea sentido como algo extraño, fuera de proporción o de lugar. Freud teorizó ampliamente el concepto de "afecto", como una de las dos facetas del 
representante pulsional. El afecto es susceptible de separarse de la parte "representación" para colocarse en otras representaciones substitutivas. El afecto, además, puede experimentar acumulación, desplazamiento y/o descarga. La posibilidad de registro de una tonalidad afectiva puede ir desde sensaciones muy vivas hasta meras "señales", puede formar parte de sentimientos elaborados o puede descualificarse bajo la forma de angustia.

Fundamental es notar que la noción de "envoltura" aparece en la obra de Freud en 1920, en el momento en que opera una reformulación de la tópica psíquica para introducir las instancias de ello, yo y superyó. Freud presenta al yo como una metáfora: el yo tiene la configuración de un saco englobante que representa la superficie del cuerpo; o también como una metonimia: el yo surge en la superficie del aparato psíquico como una entidad especializada en la relación con el mundo exterior. Destacamos la noción de "envoltura psíquica" porque el yo constituye el saco, no tanto del cuerpo, sino de la representación del cuerpo.

La idea de "yo piel", por su parte, tiene sus antecedentes en la de "piel psíquica" de Esther Bick. En 1968, esta autora propone el término para explicar que las partes de la personalidad del niño pueden ser ligadas en la medida en que este introyecta un objeto bueno continente, representado por la madre. Este objeto es experimentado como una "piel", con lo cual se instala una protección en el interior y una frontera con el exterior. A partir de ahí se organiza el mundo y se lo puede simbolizar en objetos interiores y en un objetos exteriores. Habremos de observar que seis años más tarde, Didier Anzieu propone en 1974, la noción de "yo piel". Así llama éste autor al

... entorno maternante que 'rodea' al niño con una envoltura externa de mensajes que se ajusta con cierta suavidad dejando un espacio disponible a la envoltura interna, a la superficie del cuerpo del bebé, lugar e instrumento de emisión de mensajes: ser un Yo es sentir la capacidad de emitir señales que los demás reciben [...] Esta envoltura a la medida, termina la individualización del bebé por el reconocimiento que le aporta la confirmación de su individualidad: tiene su estilo, su temperamento propio, diferente de los demás sobre un fondo de parecido. Ser un Yo es sentirse único. (Anzieu, 1985, p. 61)

Anzieu, como vemos, retoma la idea de Freud propuesta entre otros lugares en "La pizarra mágica" (1925), en donde el aparato psíquico es pensado con una doble envoltura. Siguiendo a Freud, Anzieu (1990) dice al respecto:

La envoltura psíquica comprende dos capas diferentes en su estructura y su función. La capa más externa, la más periférica, la más dura, la más rígida, está vuelta hacia el mundo exterior. Ella hace pantalla a las estimulaciones, principalmente físico químicas, mismas que provienen de ese mundo. Es la paraexcitación. La capa interna, más delgada, más suave, más sensible, tiene una 
función receptora. Ella percibe índices, señales, signos y ella permite la inscripción de sus trazas. Es a la vez una película y una interfase: una película frágil de doble cara, una vuelta hacia el mundo exterior, la otra hacia el mundo interior: una interfase entonces que separa esos dos mundos poniéndolos en relación. (p. 32)

Interesante es notar que tanto para Anzieu como para Freud, la intervención de la madre es la que permite el funcionamiento de esa doble capa, por un lado de protección y por otro lado de excitación, de contacto y de comunicación. El ser humano nace inmaduro, indefenso e incapaz de sobrevivir sin ayuda externa. Son los adultos, los que vuelcan sobre el bebé sus expectativas y sus deseos, quienes sirven de base para la constitución psíquica del sujeto. La persona construye su vida pulsional y las maneras de hacer frente a ella (instancias y mecanismos de defensa) en la relación con los otros adultos que le sirven de modelo y de objeto de investiduras.

Ahora bien, además de la gran importancia que representan las protecciones antiestímulo frente a las cantidades de excitación externas, el aparato psíquico dispone de una defensa específica delante de cantidades intrapsíquicas, y esta consiste en procesos de distribución de la cantidad, y es aquí donde Freud introduce la noción de yo. Este tendría la importantísima función de inhibir la descarga plena de los procesos que transitan por las huellas mnémicas de carácter displacentero. El yo comanda así los diferentes mecanismos de defensa, los cuales introducen una modificación importante al imperio del principio de placer.

En ese sentido, la teoría de Freud nos permite entender cómo a partir de la constitución narcisista, es decir de la constitución del yo de la persona, el aparato psíquico puede hacer frente a los diversos embates pulsionales y de los estímulos.

Mencionaremos en lo que sigue algunos de los elementos fundamentales desprendidos de la teoría, los cuáles constituyen nuestras herramientas del trabajo en la atención emocional en la Unidad de niños con quemaduras.

Respecto al trauma psicológico, como sabemos, se produce cuando el aparato psíquico y sus mecanismos de distribución de cantidad se ven rebasados por la recepción de estímulos de magnitud elevada, para los cuales el aparato no se encontraba preparado. A diferencia de algunos enfoques teóricos que piensan el trauma psicológico como generado por la mera situación del accidente, en psicoanálisis consideramos que todo traumatismo es producto del efecto integrado de la historia del sujeto, es decir que es el producto de series de momentos eslabonados por la "resignificación" de huellas mnémicas previas asociadas a eventos posteriores.

Con "contenimiento" por su parte, hacemos referencia a la noción aportada por el psicoanálisis británico que plantea que la madre del bebé recién nacido recibe de este las angustias caóticas y el trastorno que el bebé siente que ha 
ocurrido en su mundo. La madre recibe y pondera dichas angustias devolviéndole la versión de un mundo más ordenado y más posible de soportar y de vivir. Pensamos que esa función de contenimiento de las angustias caóticas de los niños quemados y de sus familiares es la que realiza el equipo clínico mediante el dispositivo de "acompañamiento psicológico", escuchando y comprendiendo los movimientos transferenciales y las interacciones que viven estas personas durante su proceso de hospitalización.

A efectos de nuestra investigación, hemos concebido una metodología cualitativa, con la finalidad tanto de estudiar las condiciones metapsicológicas que caracterizan estas situaciones, como de evaluar de nuestra intervención. En ese sentido trabajamos en la "Unidad de Quemados" con el dispositivo que hemos denominado "acompañamiento psicológico". Un dispositivo cuyas características se describen enseguida.

El Servicio de psicología del hospital no espera a que se formule una solicitud de interconsulta para proponerse a intervenir, sino que todo niño que llega al servicio es visitado desde que se da su ingreso. Ni el paciente en piso, ni sus familiares solicitan la visita del Servicio de psicología, de manera que cuando llegamos por primera vez hasta su cama nos identificamos, manteniéndonos atentos a los indicios de apertura y disposición a recibirnos y a hablar con nosotros.

A diferencia del dispositivo de psicoterapia individual, de pareja o de grupo, en que el usuario de los servicios junto con el terapeuta establecen un contrato con honorarios y un horario previo concertado; en nuestro dispositivo de acompañamiento psicológico el paciente y sus familiares están en el piso todo el tiempo, de manera que las visitas de nuestra parte tratan de ser regulares, frecuentes, en algún momento previamente concertadas, aunque también pueden ser espontáneas y/o realizadas en función de las necesidades del paciente y de su familia.

Llegamos hasta donde las personas nos lo permiten y están de acuerdo. Mas que interrogar acerca de "algo" que queramos saber, estamos ahí para escuchar lo que las personas tienen a bien confiarnos, decirnos o compartirnos. Partimos de la premisa de que las personas dicen más de lo que creen que están diciendo y que muchas veces quieren esclarecer sus sentimientos y preocupaciones al comunicárnoslos. Es la persona la que sabe acerca de ella misma y es la única que puede tomar sus decisiones, de modo que evitamos inclinar en algún sentido específico sus opciones de vida.

Dejamos todo el espacio posible a la asociación libre. Ello bajo la figura de entrevistas no estructuradas, preguntas abiertas, escucha atenta del paciente y de su familia, para recibir de ellos todos los materiales significantes que aporten, 
discursivos y no discursivos. Optamos por una escucha que trate de incluir también los elementos que no están explícitos claramente, pero que la persona quiera abordar.

Contribuimos al esclarecimiento de los conflictos psicológicos, en el caso en que la situación así lo permita, respetando la capacidad de comprensión de cada niño y de sus padres, evaluando su evolución, su comprensión del tratamiento y examinando los recursos personales y familiares en juego.

Acompañamos en su vivenciar a estas personas, ayudando en lo posible al contenimiento de sus angustias caóticas. El "contenimiento" es indispensable que se realice en el seno del equipo clínico. La atención de situaciones graves y de urgencia remueve y actualiza movimientos transferenciales en el personal y en los usuarios, por tanto no será posible hacerles frente convenientemente si no es en un trabajo de equipo.

Utilizamos en todo el dispositivo instrumentado, las técnicas de entrevista clínica psicológica y psicoanalítica, con análisis y sistematización de la información recabada. Así, trabajamos la mayor parte del tiempo al lado de la cama del niño quemado, junto con el familiar que lo acompaña, y en ocasiones en entrevistas por separado en un cubículo anexo, según se requiera.

Discusión. Al igual que en otro tipo de accidentes, el paciente hospitalizado por quemaduras ha vivido una situación traumática, no sólo físicamente, sino también psicológicamente. Mientras que en los accidentes con lesiones, fracturas o amputaciones hay dolor, en el caso del quemado grave el dolor se caracteriza por no presentar ningún cese o mengua ya que las barreras de protección antiestímulo (la piel) están desmanteladas en amplias zonas.

Si bien en la terapéutica de hoy el dolor puede ser apaciguado momentáneamente con anestésicos y analgésicos eficaces, el paciente quemado está desprotegido ante los estímulos más cotidianos sintiéndolos como una agresión a su integridad corporal: cuando la sábana toca la herida esta se puede adherir y arrancar parte de la piel que el organismo intenta regenerar; el paciente puede sentir frío aunque su cuarto aislado tenga la temperatura elevada mediante calefacción; el movimiento de una articulación abre con facilidad la costra que después de unos días ha conseguido formarse en la superficie quemada y la hace sangrar de nuevo.

Conocemos múltiples autores que han descrito la manera como un adulto puede sufrir y hacer frente al trauma psicológico de un accidente, en particular por quemaduras.

Reacciones emocionales observadas en los niños quemados y en sus familias. En la relación transferencial con los otros, el dolor se vuelve insoportable, o por el contrario más tolerable. Las conductas manifiestas muestran que los niños soportan mejor el dolor cuando son acompañados por algún familiar más que por otro. Hay mamás o papás más comprensivos y suaves de trato que 
otros, sin embargo, los cuidados, las curaciones o la alimentación de un niño, realizados con calidad semejente por el adulto, tienen para el niño efectos muy diversos en función de la relación transferencial vigente.

En efecto, el dolor en el niño se vuelve soportable, incluso de manera heroica, al ser sostenido por una relación de apoyo y de confianza que encuentra su enclave en la historia libidinal del sujeto.

Por otra parte, los efectos terapéuticos en contra del dolor que ejercen los elementos lúdicos, las investiduras de interés y de entretenimiento son evidentes. El tratamiento lúdico de una situación permite que el niño recree su realidad organizándola de modo menos amenazante y más amable. En esta realidad recreada el niño coloca a los objetos peligrosos bajo control, monta poderosos mecanismos de protección y dispone de amplios márgenes de maniobra anticipatoria del peligro. En suma, el niño cuenta con una "realidad" confiable que le ayuda a enfrentar la realidad de riesgos que se encuentra viviendo. El psicólogo o psicoanalista no alienta con eso ilusiones falsas o espejismos, simplemente trabaja con la gran capacidad del niño para revertir una situación sufrida pasivamente en una realidad en la que el niño se coloca como "activo" (recordemos el caso del fort-da de Freud).

Ahora bien, aunque la expresión afectiva y el procesamiento emocional no se produce exclusivamente mediante la palabra, esta última constituye el elemento por excelencia para desgastar el efecto traumático de las experiencias vividas y resentidas. Las palabras permiten abrir perspectiva cuando la persona no ve más allá de su momento de sufrimiento. Las palabras aportan oxígeno cuando la persona se ahoga en su angustia. Las palabras conectan el presente con el pasado y con el futuro, aportando con ello la temporalidad. No es raro encontrar a niños quemados que demandan que les hablen "porque les duele mucho" o que una vez que se han animado con una conversación puedan olvidarse un poco de su dolor.

Por su parte, en escenarios en que se atienden situaciones muy graves y se prodigan cuidados que causan dolor, como es el caso de la Unidad de Quemados, las fantasías de "estar siendo torturado", de "estar siendo desollado", de "no estar siendo bien atendido, por negligencia o por incapacidad", de "estar muriéndose", son muy frecuentes que aparezcan. Ello hace indispensable trabajar con los pacientes y sus familias para que se permitan expresarlas y trabajarlas. El pasaje entre el sentir que "se está siendo curado con los mejores cuidados aunque dolorosos" y el sentir de que "se está siendo objeto de una intervención descuidada y/o sádica" es muy fácil de darse. Se requiere estar al pendiente de esas vivencias subjetivas a fin de ofrecer la mejor atención y también de contar con la mejor disposición de parte del paciente y de sus familiares. 
Por último, los miembros del equipo clínico no están exentos tampoco de presentar este tipo de fantasías, de sentir culpa frente a ellas, o de pretender reconocerlas en otros colegas, ya que el dolor de los niños accidentados alcanza grandes proporciones. Será la calidad del procesamiento de esas fantasías y sentimientos que el equipo clínico realice la que permitirá endigarlos y tramitarlos en beneficio del trabajo de equipo y de la relación terapéutica establecida con los pacientes y sus familiares. El niño y su familia necesitan, en fin, conocer y entender el sentido de los diferentes procederes y cuidados médicos que se les aportan día con día. Buena parte de las relaciones conflictivas entre usuarios y personal del hospital, así como la no cooperación adecuada ante el tratamiento del paciente, se genera por deficiencias en la comunicación o fallas en la comprensión de la razón de los procederes médicos. El psicólogo, dado el lugar de confianza en que muchas veces es colocado, puede hacer una labor de mediación neutral y de facilitación de la comunicación entre los usuarios y el demás personal del hospital.

\section{Referencias}

AnZIeu, Didier. Le Moi-peau. Paris: Dunod, 1985.

. L'épiderme nomade et la peau psychique. Paris: Editions Apsygée, 1990.

Freud, Sigmund (1895). Proyecto de psicología. In: Obras completas. Buenos Aires: Amorrortu, 1976. v. I.

. (1920). Más allá del principio de placer. In: Obras completas. Buenos Aires: Amorrortu, 1976. v. XVIII.

. (1925). Nota sobre la "pizarra mágica”. In: Obras completas. Buenos Aires: Amorrortu, 1976. v. XIX.

\section{Resumos}

(Reações emocionais das crianças hospitalizadas com queimadura e de seus familiares)

Os acidentes por queimaduras comportam um forte caráter traumático, ele é ainda maior quando o acidentado é uma criança. O presente texto expõe o trabalho de acompanhamento psicológico e psicanalítico desenvolvido na unidade de queimados do Hospital Civil de Guadalajara. São apresentados os aspectos teóricos e 
metodológicos que servem de base para a intervenção, podendo constatar a importância da relação transferencial, dos aspectos lúdicos, da livre expressão da afetividade e do trabalho da equipe como fatores redutores do impacto traumático do acidente.

Palavras-chave: Trauma psíquico, dor, transferência, brincar parents)

(Comportements émotionnels des enfants brûlés hospitalisés ainsi que de leurs

Les traumatismes liés aux brûlures accidentelles sont d'autant plus sévères quand la victime est un enfant. Dans ce travail, il s'agira de rendre compte d'un travail d'accompagnement psychologique et psychanalytique effectué au Service des Brûlés de l'Hôpital Civil de Guadalajara. Les aspects théoriques et méthodologiques servant de base à cette intervention y seront analysés, de même que l'importance du rapport de transfert, des éléments ludiques, de la libre expression des affects et du travail d'équipe dans la réduction de l'impact traumatique.

Mots clés: Trauma psychique, douleur, transfert, jeu families)

(Children hospitalized with burns: their emotional reactions and those of their

Persons who undergo accidental burning suffer severe traumatic effects, all the more so when these victims are children. This article deals with the psychological and psychoanalytic task of accompanying such persons at the Burned Patients Unit at the Guadalajara Civil Hospital, in Mexico. The theoretical and methodological aspects upon which the intervention is based is then discussed, as well as the importance of the transferential relationship, play activities, the free expression of affects and the teamwork involved in reducing the traumatic impact of the accident.

Key words: Psychic trauma, pain, transferential relationship, play

Versão inicial recebida em dezembro de 2006 Versão aprovada para publicação em outubro de 2007

\section{Gabriel Zárate Guerrero}

Doctor en Estudios Psicoanalíticos, Université Paris 7; profesor investigador de tiempo completo en el Centro Universitario de Ciencias de la Salud (CUCS), de la Universidad de Guadalajara (México).

Calle Monte Olimpo No. 1515 Colonia Independencia

C.P. 44340 Guadalajara Jalisco, México

Teléfono (33) 36096838 .

e-mail: zarate@ cucs.udg.mx 